\title{
Acesso a PrEP por homens cisgênero e transexuais: Um estudo de abordagem qualitativa
}

\author{
Access to PrEP by cisgender mens and transsexual person: A qualitative study \\ Acceso a la PrEP por hombres cisgénero y transexuales: Un estudio cualitativo
}

Recebido: 10/01/2021 | Revisado: 12/01/2021 | Aceito: 18/01/2021 | Publicado: 24/01/2021

Marcus Vinicius Lessa de Souza ORCID: https://orcid.org/0000-0002-3023-4778

Universidade Veiga de Almeida, Brasil

E-mail: contato.marcuslessa@gmail.com

Roni Robson da Silva

ORCID: https://orcid.org/0000-0001-6010-6438

Universidade Veiga de Almeida, Brasil

E-mail: rr.roni1 @gmail.com

Maria Cristina Pimenta de Oliveira

ORCID: https://orcid.org/0000-0002-4205-9786

Fundação Oswaldo Cruz, Brasil

E-mail: profmcrispimenta@uol.com.br

Leandro Andrade da Silva

ORCID: https://orcid.org/0000-0003-3213-5527

Universidade Veiga de Almeida, Brasil

E-mail: proflandrade@gmail.com

Maria Virginia Godoy da Silva

ORCID: https://orcid.org/0000-0003-3980-042X

Universidade Veiga de Almeida, Brasil godoydasilva@terra.com.br

Divane de Vargas

ORCID: https://orcid.org/0000-0003-3140-8394

Universidade de São Paulo, Brasil profdivanevargas@uol.com.br

Rodrigo Leite Hipólito

ORCID: https://orcid.org/0000-0002-2439-7626

Universidade Federal Fluminense, Brasil professorrlh@uol.com.br

Maria das Graças Gazel de Souza

ORCID: https://orcid.org/0000-0002-6247-9331 Instituto Nacional do Câncer, Brasil mgracagazel@uol.com.br

Maria Lucia Feitosa Goulart da Silveira ORCID: https://orcid.org/0000-0003-1716-7088 Universidade Iguaçu, Brasil mlucia@gmail.com

Lucas Marvilla Fraga de Mesquita ORCID: https://orcid.org/0000-0003-3851-7366 Universidade Estácio de Sá, Brasil lucasmfraga@gmail.com

Michael Silva Araújo

ORCID: https://orcid.org/0000-0002-5389-5712 Universidade Federal de Itajubá, Brasil michaelsilva@gmail.com Lígia Paula Ignácio

ORCID: https://orcid.org/0000-0001-6147-6764 Fundação Técnico-Educacional Souza Marques, Brasil ligiapaula@gmail.com

Tatiana Vasconcellos Fontes ORCID: https://orcid.org/0000-0003-1832-5658 Fundação Técnico-Educacional Souza Marques, Brasil tativasconcellos@gmail.com

Ícaro Ferracini de Alencar ORCID: https://orcid.org/0000-0003-3852-7767 Universidade Iguaçu, Brasil icaroferracini@gmaol.com 
Daniele Augusto Correa Souza

ORCID: https://orcid.org/0000-0002-9141-713X

Universidade Iguaçu, Brasil daniaugusto@gmail.com

João Vitor Emídio Oliveira

ORCID: https://orcid.org/0000-0002-6514-6620

Universidade Iguaçu, Brasil joaovitor@gmail.com

Milena Preissler das Neves

ORCID: https://orcid.org/0000-0002-3890-924X Universidade Veiga de Almeida, Brasil mpreeeslier@gmail.com

Amanda Vargas Pereira

ORCID: https://orcid.org/0000-0002-5953-3712 Fundação Técnico-Educacional Souza Marques, Brasil amandavergas@gmail.com

Marcelo Oliveira Soares Filho

ORCID: https://orcid.org/0000-0003-2671-9999

Centro Universitário Rio de Janeiro, Brasil marcelooliver@gmail.com

Vania de Cassia de Araujo Dutra

ORCID: https://orcid.org/0000-0001-9377-7342

Universidade de Vassouras, Brasil vaniadecassia@gmail.com

\title{
Resumo
}

Estudo realizado no Rio de Janeiro, investiga a percepção de usuários de serviços públicos que ofertam a profilaxia préexposição ao HIV. Objetivo: Levantar possíveis barreiras e fatores facilitadores no acesso à Profilaxia Pré-Exposição (PrEP). Método: Trata-se de pesquisa com abordagem qualitativa, realizada com usuários de serviços que oferecem PrEP no grande Rio, entrevistas foram gravadas e transcritas. As categorias de análise são derivadas da construção das experiências narradas pelos interlocutores. Resultados e Discussão: Foram entrevistados 12 usuários dessa terapêutica com idade entre 25 e 39 anos, sendo 10 homens que fazem sexo com outros homens e 2 mulheres trans. Todos os usuários relataram ter algum conhecimento sobre PrEP; a pessoa transexual é a que menos frequenta os serviços. Existe consenso que a prevenção combinada é um passo positivo na política de prevenção a ISTS. Conclusão: As principais barreiras dizem respeito às limitações estruturais dos serviços de saúde.

Palavras-chave: Profilaxia pré-exposição; Infecções sexualmente transmissíveis; Homens que fazem sexo com homens; Transgênero; Prevenção primária.

\begin{abstract}
A study carried out in Rio de Janeiro, investigates the perception of users of public services that offer HIV pre-exposure prophylaxis. Objective: To raise possible barriers and facilitating factors in accessing Pre-Exposure Prophylaxis (PrEP). Method: This is a research with a qualitative approach, conducted with users of services that offer PrEP in the greater Rio, interviews were recorded and transcribed. The analysis categories are derived from the construction of the experiences narrated by the interlocutors. Results and Discussion: 12 users of this therapy aged between 25 and 39 years were interviewed, 10 men who have sex with other men and 2 trans women. All users reported having some knowledge about PrEP; the transgender person is the one who attends the services least. There is a consensus that combined prevention is a positive step in the STI prevention policy. Conclusion: The main barriers are related to the structural limitations of health services.
\end{abstract}

Keywords: Pre-exposure prophylaxis; Sexually Transmitted infections; Men who have sex with men; Transgender; Primary prevention.

\section{Resumen}

Un estudio realizado en Río de Janeiro investiga la percepción de los usuarios de los servicios públicos que ofrecen profilaxis preexposición al VIH. Objetivo: Levantar posibles barreras y factores facilitadores en el acceso a la profilaxis previa a la exposición (PrEP). Método: Se trata de una investigación con enfoque cualitativo, realizada con usuarios de servicios que ofrecen PrEP en el gran Río, se grabaron y transcribieron entrevistas. Las categorías de análisis se derivan de la construcción de las experiencias narradas por los interlocutores. Resultados y Discusión: Se entrevistó a 12 usuarios de esta terapia con edades entre 25 y 39 años, 10 hombres que tienen sexo con otros hombres y 2 mujeres trans. Todos los usuarios informaron tener algún conocimiento sobre PrEP; la persona transgénero es la que menos asiste a los servicios. Existe consenso en que la prevención combinada es un paso positivo en la política de prevención de ITS. Conclusión: Las principales barreras están relacionadas con las limitaciones estructurales de los servicios de salud.

Palabras clave: Profilaxis previa a la exposición; Infecciones de transmisión sexual; Hombres que tienen sexo con hombres; Transgénero; Prevención primaria. 


\section{Introdução}

De acordo com o Boletim epidemiológico de HIV/AIDS publicado em 2019 de 1980 até junho de 2019 foram identificados 965.967 casos de HIV no Brasil, sendo 633.462 (65,6\%) casos em homens e 332.505 (34,4\%) em mulheres (Brasil, 2019). O país tem registrado uma média anual de 39 mil novos casos de aids nos últimos 5 anos. O número anual de casos de aids vem diminuindo desde 2013, quando atingiu 43.269 casos e em 2017 foram registrados 37.791 (Brasil, 2017; 2019). O Brasil apresenta um padrão de epidemia concentrada (Amaral et al., 2020), onde os homens gays; homens que fazem sexo com outros homens (HSH); pessoas Transexuais (Trans) e profissionais do sexo, respondem pela maioria de casos novos da infecção pelo HIV (Hipólito et al., 2020). Na categoria de faixa etária, o número de adolescentes e jovens de 15-24 anos infectados pelo HIV tem crescido consideravelmente (Silva et al., 2019). Essas pessoas frequentemente estão sujeitas a situações de discriminação, sendo alvo de estigma e preconceito, potencializando assim sua vulnerabilidade à infecção pelo HIV e a outras infecções sexualmente transmissíveis (UNAIDS, 2017).

Segundo o Protocolo Clínico e Diretrizes Terapêuticas (PCDT) criado pelo Ministério da Saúde do Brasil para Profilaxia Pré-Exposição ao HIV (PrEP) consiste no uso de medicamentos antirretrovirais (ARV) por meio de dose fixa de FTC/TDF (Entricitabina 200mg / Tenofovir 300mg) por via oral uma vez ao dia, por pessoas HIV negativas, com intuito de reduzir o risco de adquirir a infecção pelo HIV (Brasil, 2017). Estudos clínicos realizados comprovam a efetividade e eficácia da PrEP: iPrEx (Peru, Equador, África do Sul, Brasil, Tailândia, EUA, 2007-2010), PROUD (Reino Unido 2012-2014), PrEP Brasil (Brasil, 2014-2016) e IPERGAY (França e Canadá, 2015-2016) (UNAIDS, 2017). A estratégia tem se mostrado segura e eficaz em pessoas com risco elevado de adquirir a infecção (Baral et al., 2018).

Nesse contexto, no Brasil, a PrEP se insere como uma ferramenta adicional de prevenção no âmbito da prevenção combinada, disponível no Sistema Único de Saúde do Brasil (SUS) desde dezembro de 2017 (Bastos et al., 2018). A política de PrEP preconiza o uso diário dos ARVs com o objetivo de reduzir a transmissão do HIV (Carvalho \& Vergara, 2016), realizar o diagnóstico precoce do HIV e outras ISTs, o acompanhamento trimestral do usuário, o tratamento das IST, a imunização para as hepatites, e contribui para o alcance das metas relacionadas ao fim da epidemia (Grinsztejn, 2019). A PrEP auxiliará no alcance dessa meta (Silva et al., 2019).

Em relação às populações sob maior risco de infecção pelo HIV no Brasil, estudos brasileiros recentes de abrangência nacional, utilizando a metodologia Respondent-Driven Sampling (RDS), que apresentam prevalências de HIV, sífilis e hepatites B e C, HSH (Brasil, 2019; Couto, Paiva, Oliveira, Gomes, Rodrigues, \& Teixeira, 2020) travestis e transgênero, e profissionais do sexo feminino, demonstram que em mulheres transexuais existe um risco em até 49 vezes maior de adquirir o HIV, se comparado com adultos da mesma faixa etária, que pertencem a população em geral (D'Avanzo, 2020) . Segundo o Programa das Nações Unidas para a Aids (UNAIDS), as mulheres transgêneras estão entre as populações mais afetadas pelo HIV. Estimase que 19\% das mulheres transexuais estejam vivendo com HIV (Sevelius, Deutsch \& Grant, 2016). Nesse sentido, dentre as estratégias de prevenção combinada, a PrEP foi inserida em nosso país pelo Ministério da Saúde objetivando oferecer mais um instrumento de prevenção para o usuário com práticas de maior risco, a mesma estratégia foi adotada nos Estados Unidos da América em 2012 (Molina et al., 2015).

A escassez de estudos em âmbito nacional que tratem dessa temática torna a pesquisa relevante. Sendo essa população inserida em contexto de vulnerabilidade amplamente conhecido (Neves et al., 2019), ao explorar suas percepções, atitudes e conhecimentos acerca dos possíveis benefícios ofertados pela PrEP (Hipólito et al., 2020), além das possíveis barreiras enfrentadas para o acesso à profilaxia é possível contribuir para a melhoria do acesso a PrEP (Grinsztejn, 2019) e o aprimoramento da implementação das ações de prevenção combinada ao HIV, no Rio de Janeiro (Kerr et al., 2018).

O trabalho aqui proposto com os usuários de PrEP é um recorte do estudo qualitativo PrEP Stakeholders, mais abrangente, no âmbito do projeto ImPrEP que está sendo conduzido no Brasil com o objetivo geral de avaliar aspectos 
estratégicos na implementação da política de PrEP, servindo como uma etapa de preparação para a implantação de serviços integrados de PrEP no Brasil e em outros países (Meireles \& Brito 2020).

O presente estudo foi realizado com usuários em serviços que oferecem PrEP no Rio de Janeiro, para levantar suas concepções, conhecimentos, atitudes e experiências com o uso da PrEP (Silva et al., 2020). Do ponto de vista teórico, a pesquisa considera a experiência humana em termos da análise de sua ação, a intencionalidade e a inserção em contextos, como pensado por Schutz, na perspectiva das relações sociais e a interlocução entre os atores na vida cotidiana, como espaço onde se processam essas dimensões sociais (Reisner et al., 2019). O objetivo do presente estudo é investigar a percepção de usuários de serviços de saúde que ofertam PrEP, para levantar possíveis barreiras e fatores facilitadores no acesso ao PrEP por HSH e mulheres transexuais (Barbosa et al., 2020).

\section{Metodologia}

Trata-se de um estudo de abordagem qualitativa, que privilegia uma perspectiva fenomenológica (Pereira, 2018), buscando apreender as concepções e experiências de homens gays, pessoas HSH e pessoas Trans sobre o acesso e uso da PrEP no âmbito da prevenção combinada (Mendonça et al., 2020) em dois serviços de referência do Rio de Janeiro. Denominado serviço 1 é um hospital público federal, localizado na zona sul do Rio de Janeiro, já o serviço 2, é um hospital confessional localizado na região central da cidade. A análise dos dados foi desenvolvida de acordo com princípios fundamentados na análise de conteúdo de Bardin, que situa o conteúdo das narrativas no plano epistemológico e compreensivo, exprimindo os significados atribuídos pelos próprios interlocutores (Bardin, 2011).

Os critérios de inclusão dos participantes são: ser homem gay ou HSH, ou mulher Trans, idade $\geq$ a 18 anos, ser capaz de compreender e dar consentimento prévio e estar em uso de PrEP na rede pública no Rio de Janeiro. Os critérios de exclusão são: mulheres cis gênero e mulheres heterossexuais. Os participantes foram previamente esclarecidos quanto aos objetivos do estudo, e sua participação foi voluntária e assinaram o termo de consentimento livre e esclarecido (TCLE). As entrevistas foram conduzidas de forma presencial em um ambiente confortável e confidencial. As entrevistas foram gravadas em áudio, com duração de 40 a 50 minutos. O projeto foi submetido a análise ética e aprovado pelo Comitê de Ética em Pesquisa do Instituto Nacional de Infectologia Evandro Chagas - INI/Fiocruz e aprovado conforme o CAAE: 94050418.4.0000.5262.

Para a análise dos dados, o conteúdo de áudio das entrevistas foi transcrito, classificado e analisado por meio de unidades de análise do software QSR N - Vivo para análise de dados qualitativos. Os dados foram coletados no período de novembro de 2018 a abril 2019. A análise utilizou da abordagem fenomenológica acima indicada, seguindo as seguintes etapas (Carvalho \& Vergara, 2016): 1. leitura profunda e extensiva do corpus das narrativas para identificação das unidades de análise a serem codificadas com base no quadro conceitual do estudo. 2. a categorização dessas unidades de análise, a partir de sua significação nos diversos contextos, por meio de relações de similaridade e diferenças. 3. a sistematização significada do conjunto do corpus narrativo, que permite identificar o entendimento dos interlocutores sobre a implementação da política da PrEP. A organização dos dados para análise foi a partir da categorização do conteúdo das entrevistas conforme as unidades de análise apresentadas no Quadro 1. 
Quadro 1 - Categorização do conteúdo das entrevistas.

\begin{tabular}{|l|}
\hline Nós e Sub-nós \\
\hline Nós e sub-nós de Análise: \\
\hline 1. Serviços de Saúde e PrEP \\
1.1 Acesso \\
a. $\quad$ Barreiras \\
b. Facilitadores \\
\hline 2. Percepção e conhecimento sobre PrEP \\
a. População HSH \\
b. População Trans \\
\hline 3. Práticas de Prevenção e PrEP \\
a. Prevenção Combinada \\
b. PrEP \\
c. Diagnóstico e tratamento das ISTs
\end{tabular}

Fonte: Autores (2018).

\section{Resultados e Discussão}

Foram entrevistados 12 voluntários usuários de PrEP, com idades entre 25 a 39 anos. Destes, 10 se autodeclararam homens gays ou HSH e 2 mulheres Trans. Do total 6 se autodeclararam brancas e as outras 6 pardas. Quanto ao grau de escolaridade, 2 disseram possuir ensino médio incompleto, 2 ensino médio completo e 8 ensino superior completo. Sobre o estado conjugal, 2 se declararam casadas e 10 solteiras, os dados sócio demográficos são apresentados na Tabela 2. No entanto nosso estudo tem algumas limitações, houve dificuldade para entrevistá-las, pois muitas destas pessoas quando abordadas, disseram trabalhar em empregos informais ou subempregos sem possibilidades de comparecer a entrevista. $\mathrm{O}$ mesmo ocorreu com usuários HSH e Trans que se autodeclararam negras.

Para a categoria 1 - Serviços de Saúde e PrEP, tanto barreiras como fatores facilitadores foram apresentados por ambos grupos de usuários. No âmbito das barreiras estruturais os usuários citam fatores relacionados ao acesso a PrEP, tais como, número escasso de unidades de atendimento e localização dos centros que ofertam PrEP, o horário limitado de funcionamento além do tempo elevado de espera para serem atendidos. Sobre suas próprias dificuldades impera o discurso da falta de recursos financeiros para seu deslocamento ao centro de distribuição de PrEP. 
Tabela 1 - Características Sócio Demográficas.

\begin{tabular}{lll}
\hline Gênero & & \\
HSH & 10 & $83 \%$ \\
Mulher Trans & 2 & $17 \%$ \\
\hline Raça & & \\
Branca & 6 & $50 \%$ \\
Parda & 6 & $50 \%$ \\
Negra & 0 & \\
\hline
\end{tabular}

Escolaridade

\begin{tabular}{lll} 
Ensino médio incompleto & 2 & $2 \%$ \\
Ensino médio completo & 2 & $2 \%$ \\
Ensino Superior & 8 & $96 \%$ \\
\hline
\end{tabular}

Estado Conjugal

\begin{tabular}{lll} 
Casada & 2 & $17 \%$ \\
Solteira & 10 & $83 \%$ \\
& & \\
\hline Total & $\mathrm{N}=12$ & $\%$ \\
\hline
\end{tabular}

Fonte: Autores (2018).

Algumas pessoas ainda têm receio de buscar atendimento médico, pois há o medo de serem diagnosticadas com alguma enfermidade (Ryan et al.,_2020). Uma das formas de captação de novos usuários para a PrEP tem sido a propagação da informação pelos próprios usuários do serviço (Grant et al., 2019). Segundo o estudo de Reisner et al. (2019) que sugere como sendo necessária maior sensibilização e educação em saúde preventiva, uma mudança na visão e maior conscientização em relação ao conceito de prevenção. Os respectivos usuários disseminam seus conhecimentos, citam os benefícios, indicações, explicam a terceiros suas motivações em fazer uso da PrEP. Esclarecem como funciona a profilaxia, sabendo que a margem de proteção não é $100 \%$, protegendo apenas contra o HIV, havendo risco de se infectar com outras ISTs e transmiti-las a outras pessoas caso não utilize a prevenção combinada (Reisner et al., 2019).

Nessa perspectiva na pesquisa de D’Avanzo et al. (2017) as mulheres trans enfrentam barreiras únicas no acesso a serviços de saúde preventivos, como PrEP. Essas barreiras podem ser exacerbadas por maus tratos reais ou ocorridos no passado em ambientes de saúde, mas pouco se sabe sobre a relação entre a desconfiança médica e a compreensão e conhecimentos insuficientes sobre a PrEP (D’Avanzo et al., 2017), contrapondo o estudo de Kerr et al. (2018) que aponta que os usuários sentemse confortáveis no atendimento prestado pelo serviço de saúde, pois é um ambiente livre de preconceitos e discriminação, citando ser um ambiente “Gay Friendly” (Grant et al., 2019).

Onde o atendimento pode ser realizado por alguns profissionais que são homossexuais e transexuais, porém acredita que ao expandir o serviço para outras unidades de saúde esse tipo de atendimento pode não ser confortável, sendo uma barreira, para alguns HSH (Ryan et al., 2020) que são mais reservados e que prezam pela discrição, que não assumiram publicamente sua 
orientação sexual, sendo assim ter um ambiente neutro, com mais privacidade se mostra necessário para atingir a população de HSH (McCormack et at., 2016).

Para a categoria 2 - Percepção e conhecimento sobre PrEP, os usuários destacaram que sentem falta de divulgação nas mídias em geral e informação em linguagem acessível a todo tipo de público. Foi evidenciado na narrativa do usuário o incômodo com o linguajar técnico, dificultando a compreensão das informações passadas sobre o regime de uso diário dos antirretrovirais para a profilaxia, a necessidade de dar continuidade às visitas de acompanhamento e testagem para o HIV (Sevelius, Deutsch \& Grant, 2016). Segundo a pesquisa de Jalil et al. (2018) que sugere a mulher trans como uma população-chave para a prevenção do HIV. A PrEP é uma estratégia promissora e empoderadora para a prevenção do HIV entre mulheres trans, mas recomendações específicas para trans são necessárias para implementar a PrEP de maneira eficaz nessa população (Jalil et al., 2018).

Para a categoria 3 - Práticas de prevenção e PrEP, observa-se que como a informação é considerada insatisfatória, essa também traz dificuldades na compreensão sobre a forma adequada de como proceder no caso de interrupção do uso diário do medicamento (McCormack et al., 2016) a necessidade de voltar para as visitas de acompanhamento e tratamento das ISTs, como sífilis, clamídia e gonorreia (Couto, Paiva, Oliveira, Gomes \& Teixeira, 2020). Fica claro que uma das principais barreiras observadas nas narrativas dos entrevistados ainda é a presença do estigma e preconceito com essas populações.

Enquanto os usuários aguardam para serem atendidos citam o preconceito gerado com os usuários de PrEP e o pré julgamento em relação ao perfil do usuário (Baral et al., 2018). O estudo de Bastos et al (2018) sugere ser necessário que a equipe multiprofissional ao atender o interessado em PrEP evite pré julgamentos (Hipólito et al., 2020) e o olhar de censura imaginando que o usuário tenha alguma doença. Relatam como barreira o preconceito com base em paradigmas pessoais, com base na desinformação, sendo necessária uma mudança na visão das pessoas quanto ao uso da PrEP como forma de prevenção (Brasil, 2019; Meireles \& Brito, 2020). Ampliar a visão para o conceito de prevenção combinada, pois algumas pessoas não utilizam a PrEP com medo do estigma de serem comparadas com pessoas vivendo com HIV (PVHIV) ou de pensarem que pelo fato de usar a PrEP são "soropositivos" (Molina et al., 2015). Alguns usuários consideram os critérios para o fornecimento da PrEP como muito rígidos. Relatam que é necessário melhorar o acesso à medicação, ampliar os postos de distribuição, fazer com que as pessoas tenham acesso mais fácil para esse tipo de medicação (Meireles \& Brito, 2020). Ao falar de PrEP para alguns amigos, existem aqueles que nunca ouviram falar e que se beneficiariam de informação mais acessível (Silva et al., 2019)

\section{Conclusão}

O desejo de estar protegido de uma futura infecção pelo HIV é algo que motiva os usuários a uma boa adesão a PrEP, além de melhorar a qualidade de vida. Os usuários se sentem mais protegidos nas relações sexuais, porque mesmo nos casos de rompimento dos preservativos existe a sensação de segurança pelo uso da PrEP. As principais barreiras destacadas no estudo dizem respeito às limitações estruturais dos serviços de saúde para o acesso e a garantia de atendimento aos usuários, similares as dificuldades encontradas na população em geral que utiliza os serviços oferecidos pelo SUS.

No entanto, destaca-se que as dificuldades são potencializadas nesse grupo em função dos constrangimentos decorrentes dos estigmas sociais, além dos problemas decorrentes do menor poder aquisitivo do grupo que refletem no acesso aos serviços de saúde. O atendimento realizado nas unidades de saúde aos homens gays, HSH e pessoas Trans ainda é marcado muitas vezes por obstáculos, condutas inadequadas, preconceituosas e até mesmo ofensivas.

Por outro lado, a percepção positiva com relação a oferta da PrEP, prevalece como sendo uma estratégia eficaz e de grande importância para a saúde das populações que se enquadram no perfil de maior vulnerabilidade as ISTs. O Brasil, carece de estudos sobre esta temática, almeja-se que este trabalho possa contribuir para o desenvolvimento de mais pesquisas de campo, as quais possam mapear as regiões mais críticas no que diz respeito ao acesso a PrEP, afim de que sejam implementadas medidas protetivas, preventivas e para redução de danos. 


\section{Referências}

Amaral, R. T. do, Soares, R. de F. S., Silva, S. M. T. C. da, Neves, M. P. das, Silva, L. A., \& Silva, R. R. da. (2020). Identification and analysis of Garbage code occurred when filling out the Death Certificate at the Hospital Geral de Nova Iguaçu - RJ, a reality to be modified. Research, Society and Development, 9(11), e91591110658. https://doi.org/10.33448/rsd-v9i11.10658

Baral, S., Poteat, T., Strömdahl, S., Wirtz, A., Guadamuz, T., \& Beyrer, C. (2018). Worldwide burden of HIV in transgender women: a systematic review and meta-analysis. The Lancet Infectious Diseases. 13 (3), 214-222. Recuperado de: https://www.thelancet.com/journals/laninf/article/PIIS1473-3099(12)70315$8 /$ fulltext

Barbosa, I. de S. F., et al. (2020). Care for people with a problem related to alcohol abuse: an integrative literature review study. Research, Society and Development, $9(11)$, e52091110062. https://doi.org/10.33448/rsd-v9i11.10062

Bardin, L., (2011). Análise de Conteúdo. Editora Almedina

Bastos, F., Bastos, L., Coutinho, C., Toledo, L., Mota, J., \& Velasco-de-Castro, C. (2018). HIV, HCV, HBV, and syphilis among transgender women from Brazil. Medicine. (97) S16-S24. https://journals.lww.com/md-journal/Fulltext/2018/05251/HIV,_HCV,_HBV,_and_syphilis_among_transgender.7.aspx\#R6-7

Brasil. Ministério da Saúde. (2019) Departamento de Doenças de Condições Crônicas e Infecções Sexualmente Transmissíveis. Boletim epidemiológico HIV/Aids 19. http:// http://www.aids.gov.br/pt-br/pub/2019/boletim-epidemiologico-de-hivaids-

Brasil. Ministério da Saúde. (2017). Protocolo Clínico e Diretrizes Terapêuticas para Profilaxia Pré-Exposição (PrEP) de Risco à Infecção pelo HIV. http://www.aids.gov.br/pt-br/pub/2017/protocolo-clinico-e-diretrizes-terapeuticas-para-profilaxia-pre-exposicao-prep-de-risco

Carvalho, J. L. F., \& Vergara, S. C. (2016). A fenomenologia e a pesquisa dos espaços de serviços. Revista de Administração de Empresas. 42 (3), 1-14. http://dx.doi.org/10.1590/S0034-75902002000300008

Couto, P. L. S., Paiva, M. S., Oliveira, J. F., Gomes, A. M. T., Rodrigues, L. S. A., \& Teixeira, M. A. (2020). Dilemmas and challenges for HIV prevention in representations of young Catholics. Online Brazj Nurs, 17 (1), 97-108. http://www.objnursing.uff.br/index.php/nursing/article/view/5909

D'avanzo, P. A., Bass, S. B., Brajuha, J., Gutierrez-Mock, L., Ventriglia, N., \& Wellington, C. (2020). Mistrust And Prep Perceptions Between Women Transgender: A Cluster Analysis. Behavioral Medicine (45) 2 143-152. 10.1080 / 08964289.2019.1585325

Grant, R., Lama, J., Anderson, P., McMahan, V., Liu, A., \& Vargas, L. (2019). Preexposure Chemoprophylaxis for HIV Prevention in Men Who Have Sex with Men. New England Journal of Medicine, 363 (27) 2587-2599. https://www.nejm.org/doi/full/10.1056/NEJMoa1011205

Grinsztejn, B. (2019). Retention, engagement, and adherence to pre-exposure prophylaxis for men who have sex with men and transgender women in PrEP Brasil: Lancet HIV. (5) 2 136-145. https://www.thelancet.com/journals/lanhiv/article/PIIS2352-3018(18)30008-0/fulltext. 10.1016/S2352-3018(18)30008-0

Hipólito, R. L., Oliveira, D. C. de, Cecilio, H. P. M., Marques, S. C., Flores, P. V. P., Costa, T. L. da, \& Lima, F. O. de. (2020). Quality of life of people living with HIV and their multifactorial relationships. Research, Society and Development, 9(7), e82973749. https://doi.org/10.33448/rsd-v9i7.3749

Jalil, E. M., Grinsztejn, B. G. J., Velasque, L., Makkeda, A. R., Luz, P. M., \& Moreira, R. I. (2018). Awareness, Willingness, and PrEP Eligibility Among Transgender Women in Rio de Janeiro, Brazil; JAIDS Journal of Acquired Immune Deficiency Syndromes (79) 4. https://pubmed.ncbi.nlm.nih.gov/30142140/

Kerr, L., Kendall, C., Guimarães, M. D. C., Mota, R. S., Veras, M. A., \& Dourado, I. (2018). HIV prevalence among men who have sex with men in Brazil. Medicine.(97) S9-S15. https://journals.lww.com/mdjournal/Fulltext/2018/05251/HIV_prevalence_among_men_who_have_sex_with_men_in.11.aspx

McCormack, S., Dunn, D. T., Desai, M., Dolling, D. I., Gafos, M., \& Gilson, R. (2016). Pre-exposure prophylaxis to prevent the acquisition of HIV-1 infection (PROUD): effectiveness results from the pilot phase of a pragmatic open-label randomised trial. The Lancet (387) P53-60 https://www.thelancet.com/journals/lancet/article/PIIS0140-6736(15)00056-2/fulltext

Meireles, J. V. C., \& Brito, M. de V. (2020). Imunossenescência precoce na infecção por HIV: efeito da persistência viral crônica ou da terapia antirretroviral. Research, Society and Development, 9(9), e592997436. https://doi.org/10.33448/rsd-v9i9.7436

Mendonça, E. T. M., Araújo, E. da C., Botelho, E. P., Polaro, S. H. I., \& Gonçalves, L. H. T. (2020). Vivência de sexualidade e HIV/AIDS na terceira idade. Research, Society and Development, 9(7), e483974256. https://doi.org/10.33448/rsd-v9i7.4256

Molina, J., Capitant, C., Spire, B., Pialoux, G., Cotte, L., \& Charreau, I. (2015). On-Demand Preexposure Prophylaxis in Men at High Risk for HIV-1 Infection. New England Journal of Medicine 373 (23), 2237-2246. https://www.nejm.org/doi/full/10.1056/NEJMoa1506273

Neves, M. P., Silva, R. R., Silva, L. A. da ., Silva, M. V. G. da ., Silva, M. M. dos S. da ., Francisco, M. T. R. ., \& Marta, C. B. (2020). Adição à drogas, o consumo de substâncias psicoativas por jovens, utilizando o instrumento assist. Saúde Coletiva (Barueri), 9(51), 1913-1919. https://doi.org/10.36489/saudecoletiva.2019v9i51p1913-1919

Pereira, A. S. (2018). Metodologia da pesquisa científica. UAB/NTE/UFSM.

Reisner, S. L., Moore, C. S., Asquith, A., Pardee, D. J., Sarvet, A., \& Mayer, G. (2019). High risk and low uptake of pre-exposure prophylaxis to prevent HIV acquisition in a national online sample of transgender men who have sex with men in the United States. J Int AIDS Soc. 22 (9), e25391. Recuperado de: https://doi.org/10.1002/jia2.25391

Ryan, K. E., Mak, A., Mark. S., Price, B., Fairley, C. K., \& Ruth, S. (2020). Protocol For An Hiv Pre-Exposure Prophylaxis (Prep) Population Level Intervention Study In Victoria Australia: The Prepx Study; Public Health. 5 (8) Https://Doi.Org/10.3389/Fpubh.2018.00151 
Research, Society and Development, v. 10, n. 1, e44310111843, 2021

(CC BY 4.0) | ISSN 2525-3409 | DOI: http://dx.doi.org/10.33448/rsd-v10i1.11843

Sevelius, J. M., Deutsch, M. B., \& Grant, R. (2016). The future of PrEP among transgender women: the critical role of gender affirmation in research and clinical practice. J Int AIDS Soc. (2), 2-3. Recuperado de: https://doi.org/10.7448/IAS.19.7.21105

Silva, R. R., Silva, L. A, Silva, M. V. G., Neves, M. P., Silva, M. M. S., \& Francisco, M. T. R. (2019). Os Impactos do Chemsex na saúde pública mundial: um estudo sobre uma perigosa prática sexual entre homens. Rev Saúde Coletiva Barueri. (2) 51 1920-5. https://doi.org/10.36489/saudecoletiva.2019v9i51p19201925 .

Silva, R.R., Neves, M. P., Silva, L. A., Silva, M. V. G., Hipolito, R. L., \& Marta, C. B. (2020). Consumo de drogas psicoativ as em contexto sexual entre homens gays como fator de risco para transmissão de HIV/Aids. Glob Acad Nurs. 1(3):e57. https://dx.doi.org/10.5935/2675-5602.20200057

UNAIDS - Joint Programme on HIV/AIDS. (2017). Declaração de Paris. Acabar com a epidemia da AIDS: Cidades acelerando a resposta. https://unaids.org.br/wp-content/uploads/2015/12/Declara\%C3\%A7\%C3\%A3o-de-Paris-PORT-1.pdf. 